\title{
Identifying Training Deficiencies in Military Pilots by Applying the Human Factors Analysis and Classification System
}

\author{
Wen-Chin Li \\ Psychology Department, National Defense University, Taipei City, Taiwan (Republic of China)
}

\section{Don Harris}

HFI Solutions, UK

Without accurate analysis, it is difficult to identify training needs and develop the content of training programs required for preventing aviation accidents. The human factors analysis and classification system (HFACS) is based on Reason's system-wide model of human error. In this study, 523 accidents from the Republic of China Air Force were analyzed in which 1762 human errors were categorized. The results of the analysis showed that errors of judgment and poor decision-making were commonly reported amongst pilots. As a result, it was concluded that there was a need for military pilots to be trained specifically in making decisions in tactical environments. However, application of HFACS also allowed the identification of systemic training deficiencies within the organization further contributing to the accidents observed.

accident investigation error analysis human error

human factors analysis and classification system (HFACS) training development

\section{INTRODUCTION}

\subsection{Rationale for the Study}

The continual assessment of the training curricula of military pilots is essential for maintaining safe and effective operations. Training needs do not remain static. They change as a result of an evolution in operational requirements and the introduction of new equipment and procedures. Regular analysis of these requirements and revision of training systems are required.

All training development proceeds through a series of generic stages. The interservice procedures for instructional systems development (IPISD) have arguably become the benchmark approach for training design [1]. The IPISD process has five major functional phases: analyze, design, develop, implement and control. The ana- lyze phase, upon which this research focuses, provides the foundations upon which the subsequent training design process is based. It commences with a training needs analysis (TNA) to identify important gaps between the current levels of skills, knowledge and ability and those required to meet the requirements of the task.

There are two generic types of analysis that help to identify such training needs. The first type of analysis is best characterized as a conventional form of task analysis that breaks down a task into a series of tasks and subtasks (e.g., using hierarchical task or cognitive task analysis) along with their required performance standards. This type of analysis adopts a system perspective and describes the tasks that have to be accomplished in a logical fashion [2]. The second type of analysis focuses on errors in task performance. This can be done either

Correspondence should be sent to Don Harris, Faculty of Engineering and Computing, Coventry University, Priory Street, Coventry CV1 5FB, United Kingdom. E-mail: don.harris@ coventry.ac.uk. 
at individual or group level, whereby errors can be aggregated so that common deficits in task performance can be identified. In this way, a feedback loop is established between the evaluation of performance and the development of training to remedy such weaknesses [3]. This analytic approach can take place in various ways but accidents, incidents and near misses are often analyzed to provide a rich source of information for subsequent revisions in training $[4,5]$. These data can then be used to scrutinize the types of error made so that appropriate training can be devised. Patrick suggested that training was ineffective if the linkage between the operational and training environments was degraded [3]. Conversely, well targeted, operationally-relevant training has been demonstrated to have substantial benefits.

This study applies the human factors analysis and classification system (HFACS) [5, 6, 7, 8, 9, $10,11,12,13]$ for analyzing accident data from the Republic of China (RoC) Air Force as an initial stage in the TNA process.

\subsection{HFACS}

As aircraft have become increasingly more reliable, human performance has played a proportionately increasing role in the causation of accidents. Dekker has argued that human error is systematically connected to features of peoples' tools and tasks, and also their operational and organizational environment [14]. In recent years, the focus on error in aviation accidents has shifted away from technical skill deficiencies and toward the nontechnical (or soft) skills that underpin effective crew resource management (CRM), such as decision-making, attitudes, supervisory factors and organizational culture $[15,16,17]$.

There are many human error frameworks and accident investigation schemes $[6,18,19,20,21$, 22]. Such human error classification methods are used throughout aviation to help understand and mitigate the causes of poor human performance, including being used to identify training requirements. HFACS is perhaps the most frequently used accident classification schemes in the aviation domain. HFACS is based on Reason's model of human error [4]. Active failures, which are associated with the performance of front-line operators in complex systems, and latent failures, which lie dormant within the system for a long time, serve to combine together with other factors to breach a system's defenses. According to Reason, complex systems are designed, operated, maintained and managed by human beings [23]. As a result, it is not surprising that human decisions and actions at an organizational level are implicated in all accidents. Active failures of operators have a direct impact on the safety of the systems; however, latent failures are spawned in the upper levels of organizations and are related to management and regulatory structures, including the provision and oversight of training. Wiegmann and Shappell developed HFACS based upon Reason's organizational model of error [5, $6,7,8,9,10,11,12,13]$. The analysis framework was originally designed and developed as a generic human error framework for investigating and analyzing human error accidents in U.S. military aviation operations [7]. The same authors later demonstrated its applicability to the analysis of accidents in U.S. commercial [7, 8, 9, 11] and general aviation $[5,13]$. They claim that HFACS bridges the gap between theory and practice by providing safety professionals with a theoretically based tool for identifying and classifying human errors [10]. The system focuses on both latent and active failures and their inter-relationships and, by doing so, it facilitates identifying the underlying causes of human error.

HFACS examines human error in flight operations at four levels. Each higher level is assumed to affect the next downward level in the HFACS framework (Figure 1).

- Level 1 , unsafe acts of operators (active failures), is where most causes of accidents in investigations are focused. Such causes can be classified into the two basic categories of errors and violation.

- Level 2, preconditions for unsafe acts (latent/ active failures), addresses the latent failures within the causal sequence of events as well as more obvious active failures. It also describes the context of substandard conditions of operators and the substandard practices they adopt. 

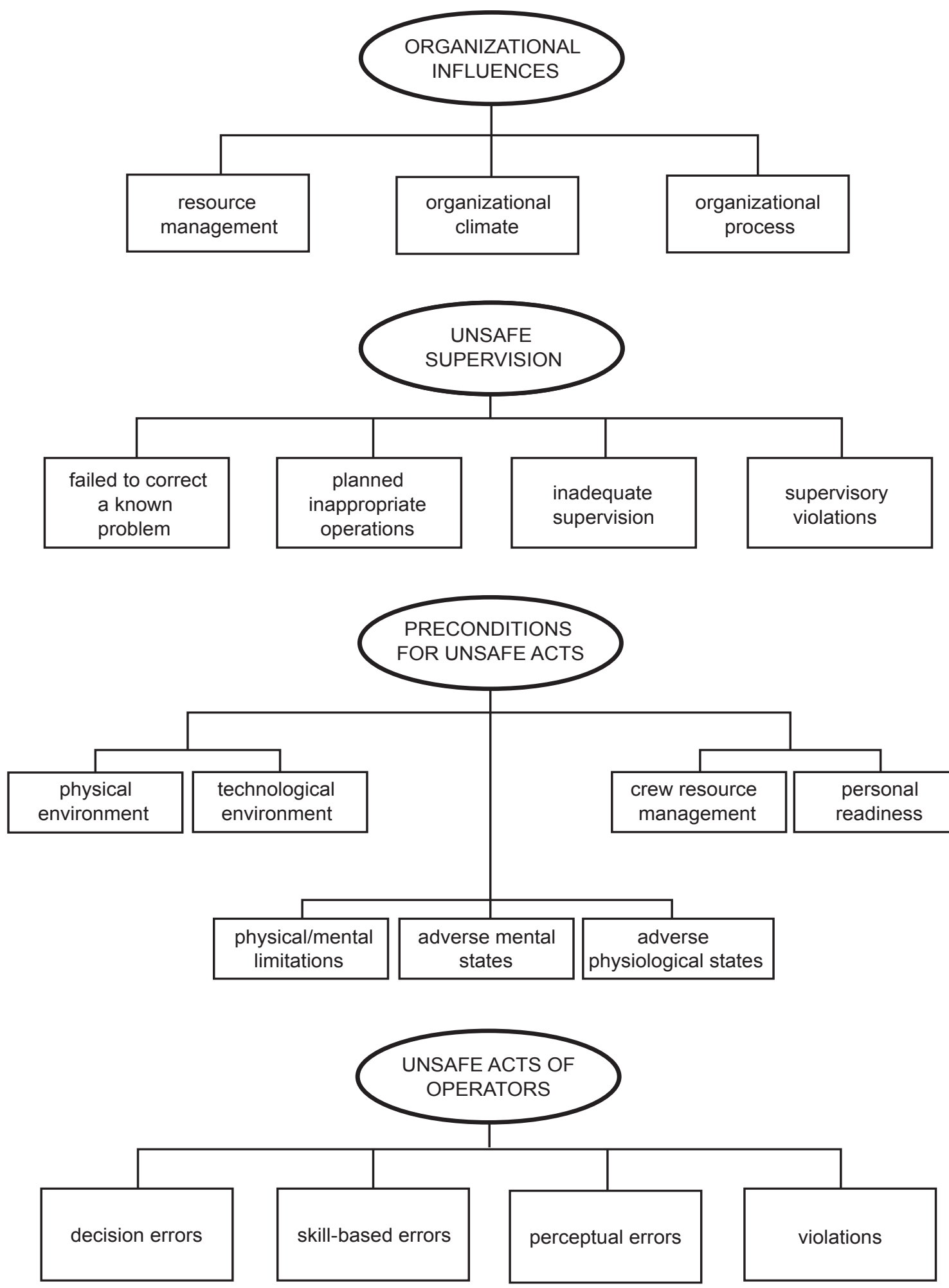

Figure 1. The human factors analysis and classification system (HFACS) framework [6].

- Level 3, unsafe supervision (latent failures), traces the causal chain of events producing unsafe acts up to the front-line supervisors.

- Level 4, organizational influences (latent failures), encompasses the most elusive of latent failures, fallible decisions of upper levels of management which directly affect supervisory practices and indirectly affect the actions of front-line operators. 
At level 1 (unsafe acts of operators), the four categories in the framework represent the physical/ mental activities of an individual that fail to achieve the intended outcomes. These are skilledbased errors, decision errors, perceptual errors and violations (Figure 1). Violations refer to the willful disregard for the rules and regulations that provide for the safety of flight. Routine violations tend to be habitual by nature and are often tolerated by the governing authority. Exceptional violations appear as isolated departures from authority, and are not necessarily indicative of an individual's typical behavior pattern, nor condoned by management [4].

Nevertheless, simply focusing on the unsafe acts of operator, the active failures immediately associated with the accident, is like focusing on a fever without understanding the underlying illness causing it. Wiegmann and Shappell classified preconditions for unsafe acts into seven subcategories of adverse mental states, adverse physiological states, physical/mental limitations, CRM, personal readiness, physical environment and technological environment [6]. Most of these subcategories, with the exception of those referring to environmental factors, can be regarded as what Reason described as psychological precursors for unsafe acts [4]. Of these categories, CRM has been regarded as a key factor in aviation safety for almost 30 years and has been the target of a great deal of research and development and is the principle area of nontechnical skills training in the aviation industry. The Joint Airworthiness Authorities defined CRM as "the effective utilisation of all resources (e.g. crewmembers, aeroplane systems and supporting facilities) to achieve safe and efficient operation" [24]. CRM evolved as an operating concept after a series of accidents in which the aircraft involved had no major technical failures (if any at all). The principal causes of these accidents were a failure to utilize all the human resources available on the flight deck in an appropriate manner. Van Avermaete described three dimensions to effective CRM: crew co-operation (team building and maintaining, considering others, supporting others and solving conflicts); leadership and managerial skills (using authority/assertiveness, pro- viding and maintaining standards, planning and coordinating, and managing workload) and situation awareness (system awareness, environmental awareness and anticipation) [25]. All of these issues are recognizable within the HFACS framework. Training in flight deck management skills produces significant beneficial changes in crew behavior [26]. After the introduction of such a program, Diehl reported an $81 \%$ drop in the accident rate in U.S. military aviation [27]. Aeronautical decision-making has also been found to be trainable $[28,29,30,31]$ and such training produces significantly better decisions in pilots [32, 33].

The role of supervisors is to provide their personnel with the facilities and capability to succeed and to ensure the job is done safely and efficiently. Level 3 in HFACS is primarily concerned with the supervisory influence both on the condition of pilots and the operational environment. HFACS contains four categories of inadequate supervision, planned inappropriate operation, failure to correct a known problem and supervisory violation (Figure 1). The provision of training is a key factor at this level. In the context of inadequate supervision, Shappell and Weigmann stated that "the supervisor, no matter at what level of operation, must provide guidance, training opportunities, leadership, and motivation, as well as the proper role model to be emulated" (p. 9) [8]. The provision and revision of training is also a factor in the other level-3 category of failure to correct a known problem. This category includes failure to provide remedial training where previously identified as necessary. This is an issue in training oversight which is a key supervisory role.

The corporate decisions about resource management are based on two conflicting objectives, the goal of safety and the goal of on-time and cost-effective operations. It needs to be noted, though, that the decisions of upper-level management can affect supervisory practices, as well as the conditions and actions of operators. Wiegmann and Shappell identified these elusive latent failures as failures in resource management, organizational climate and organizational process at level 4 in HFACS [5]. Resource management encompasses the provision of equipment, 
personnel and facilities to provide appropriate training; organizational process includes maintenance and oversight of operational standards.

Wiegmann and Shappell reported that the framework as a whole had an inter-rater reliability figure (using Cohen's $\kappa$ ) of .710, indicating substantial agreement; however, they reported no figures for the individual HFACS categories [9]. $\mathrm{Li}$ and Harris conducted further research and found the inter-rater reliabilities for the individual categories in the HFACS framework ranged between $\kappa$ values .440 and .826 [34]. Fourteen HFACS categories exceeded a $\kappa$ of .600 , which indicated substantial agreement between raters; only four categories had $\kappa$ values between .400 and 0.590 merely suggesting moderate levels of agreement [35]. Expressed as simple percentage agreement, HFACS has shown inter-rater reliabilities well in excess of $70 \%$ [7, 34, 36, 37]. As a result, it can be concluded that the coding of data using the HFACS framework is quite reliable.

\subsection{Aims and Objectives}

This study applies HFACS to analyzing human factors accident data from the RoC Air Force. The objective was to identify areas for the improvement in training provision and the associated organizational infrastructure to help mitigate the instance of human error in military aviation. To do this, it was necessary to understand the associations between errors with pilots' tools (aircraft) and tasks (flight stages). Pilots experience was assessed by their rank.

\section{METHOD}

\subsection{Accident Data}

The data set was derived from the narrative descriptions of 523 accidents from 25 years of accidents occurring in the RoC Air Force. There were 206 class- 1 accidents (cost to repair over $65 \%$ of original price or crew fatality), 78 class- 2 accidents (cost to repair of 35-64\% of original price or crew sustained serious injury) and 239 class-3 accidents (cost to repair of 3-34\% of original price or crew member sustained minor injury).

\subsection{Accident Investigation in the RoC Air Force}

The Aviation Safety Unit is responsible for all RoC Air Force accident investigations. For every accident involving a military aircraft, the investigator-in-charge follows a standard procedure for conducting the investigation. The initial stage collects all the relevant information for further analysis including identification details, pilots' information, personnel involved, aircraft information, mission and flight details, history of the flight, impact and postimpact information, meteorological information, radar information and transmissions to and from tactical air traffic control. The wreckage of the aircraft is then recovered for investigation by the engineering team. The final report details the causal factors of the accident and contains recommendations for accident prevention.

\subsection{Coding Process}

This study used Wiegmann and Shappell's version of the HFACS framework [6]. Each accident report was coded independently by two investigators, an instructor pilot and an aviation psychologist. These investigators were trained on the HFACS framework together for $10 \mathrm{~h}$ to ensure that they achieved a detailed and accurate understanding of the HFACS categories. The contents of the course included an introduction to the HFACS framework, an explanation of the definitions of the four different HFACS levels and a further detailed description of the content of each of the 18 HFACS categories. To develop a common understanding of the coding process and the HFACS categories to achieve a high level of inter-rater reliability, the two raters also jointly analyzed 2 years of $\mathrm{RoC}$ accident data prior to undertaking the main analysis.

For the purposes of analysis, aircraft were categorized as either fighter, cargo or training aircraft. Pilot's ranks were cadet pilots, second lieutenants, first lieutenants, captains, majors, lieutenant colonels (and above). Flight phases were categorized as taxi before take-off, take-off, climb-out, in the operational area; descent, approach, landing and taxi after landing. 
The presence (coded 1) or absence (coded 0 ) of each HFACS category was assessed in each accident narrative. To avoid the over-representation of a category from a single accident, each HFACS category was counted a maximum of only once per accident. Thus, the count acted simply as an indicator of the presence or absence of each of the 18 categories in a given accident.

\section{RESULTS}

In the following analyses, the percentages refer to the percentage of times that an HFACS factor was implicated in the sequence of events leading up to an accident. However, in most instances, many more than just a single factor was implicated in the accident sequence, hence, the percentages add up to over $100 \%$ across section 3 .

A total of 1762 instances of human error were recorded with the HFACS framework. Overall, unsafe acts of operators (HFACS level 1) were involved in $725(41.1 \%)$ of instances, preconditions for unsafe acts (level 2) was as a causal factor in $552(31.3 \%)$ cases, categories at unsafe supervision (level 3) were involved in 221 $(12.5 \%)$ of instances and categories at the organizational influences level (level 4) were a factor in $264(15 \%)$ accidents.

The data were cross-tabulated to describe the strength of association between the HFACS categories and aircraft type, type of mission and flight Phase. $\chi^{2}$ analyses were performed to estimate the statistical strength of association between the categories. Within each analysis, the adjusted standardized residual was computed for each cell to establish which cells within the table were significantly over- (or under-) represented.

\subsection{HFACS Categories Versus Aircraft Types}

Fighter aircraft were involved in $353(67.5 \%)$ accidents, training aircraft in $113(21.6 \%)$ accidents and cargo aircraft in 57 (10.9\%) accidents.

At level 1 (unsafe acts of operators), there were no significant associations with respect to aircraft type. At level 2 (preconditions for unsafe acts), the association of aircraft type with adverse men- tal states, CRM and personal readiness was significant. Training aircraft pilots were over-represented in having adverse mental states and personal readiness as causal factors. Cargo aircraft pilots were over-represented in having CRM problems as the major causal factor. At level 3 (unsafe supervision), the association of aircraft type with both inadequate supervision and failed to correct a known problem was significant. Training aircraft were over-represented in both these two categories. Finally, at level 4 (organizational influences), the association of aircraft type with organizational process was significant. Training aircraft pilots were again over-represented in having the organizational process category as a factor (see Tables 1-2 and Figure 2).

\subsection{HFACS Categories Versus Pilots' Rank}

Cadet pilots were involved in $30(5.7 \%)$ accidents, second lieutenants in $10(1.9 \%)$ accidents, first lieutenants in $92(17.6 \%)$ accidents, captains in $144(27.5 \%)$ accidents, majors in 148 (28.3\%) accidents and personnel with the rank of lieutenant colonels (or above) were involved in 70 (13.4\%) accidents.

At level 1 (unsafe acts of operators), decision errors, skill-based errors and perceptual errors were all significantly associated with the rank of the pilot involved in the accident. In all these categories, pilots with the rank of first lieutenant were over-represented. Cadet pilots were also over-represented in the categories of decision errors and skill-based errors. Similarly, at level 2 (preconditions for unsafe acts), first lieutenants were over-represented in having adverse mental states and physical/mental limitations as contributory factors in their mishaps. The rank of lieutenant colonel (or above) was significantly associated with causal factors in the physical environment category. At level 3 (unsafe supervision), inadequate supervision was a factor significantly over-represented in accidents involving cadet pilots and first lieutenants. Similarly, at level 4 (organizational influences), organizational process was more likely to be linked to accidents involving cadet pilots (see Tables 1 and 3, and Figure 3). 
TABLE 1. Significant $\left(x^{2}\right)$ Associations Between HFACS Categories and Type of Aircraft, Pilot's Rank and Flight Phase

\begin{tabular}{|c|c|c|c|c|}
\hline Category & Level & Type of Aircraft & Pilot's Rank & Flight Phase \\
\hline Organizational process & 4 & $x^{2}=7.74, d f=2, p<.02$ & $x^{2}=11.1, d f=5, p<.05$ & ns \\
\hline Organizational climate & 4 & $n s$ & $n s$ & $n s$ \\
\hline $\begin{array}{l}\text { Resource } \\
\text { management }\end{array}$ & 4 & $n s$ & $n s$ & $n s$ \\
\hline Supervisory violation & 3 & ns & $n s$ & $n s$ \\
\hline Fail to correct problem & 3 & $x^{2}=20.69, d f=2, p=.00$ & $n s$ & ns \\
\hline $\begin{array}{l}\text { Plan inadequate } \\
\text { operation }\end{array}$ & 3 & $n s$ & $n s$ & ns \\
\hline $\begin{array}{l}\text { Inadequate } \\
\text { supervision }\end{array}$ & 3 & $x^{2}=8.28, d f=2, p<.01$ & $x^{2}=26.6, d f=5, p=.00$ & $x^{2}=34.6, d f=8, p=.00$ \\
\hline $\begin{array}{l}\text { Technology } \\
\text { environment }\end{array}$ & 2 & $n s$ & $n s$ & $n s$ \\
\hline Physical environment & 2 & $n s$ & $x^{2}=15.1, d f=5, p<.01$ & $n s$ \\
\hline Personal readiness & 2 & $x^{2}=9.58, d f=2, p<.01$ & $n s$ & $n s$ \\
\hline CRM & 2 & $x^{2}=8.35, d f=2, p<.01$ & $n s$ & $x^{2}=19.6, d f=8, p<.01$ \\
\hline $\begin{array}{l}\text { Physical/mental } \\
\text { limitation }\end{array}$ & 2 & $n s$ & $x^{2}=32.5, d f=5, p=.00$ & $x^{2}=17.5, d f=8, p<.02$ \\
\hline $\begin{array}{l}\text { Adverse physiological } \\
\text { state }\end{array}$ & 2 & $n s$ & $n s$ & $n s$ \\
\hline Adverse mental states & 2 & $x^{2}=7.55, d f=2, p<.02$ & $x^{2}=18.3, d f=5, p=.00$ & $x^{2}=25.7, d f=8, p=.00$ \\
\hline Violations & 1 & $n s$ & $n s$ & $n s$ \\
\hline Perceptual errors & 1 & $n s$ & $x^{2}=12.5, d f=5, p<.02$ & $n s$ \\
\hline Skilled-based errors & 1 & $n s$ & $x^{2}=18.1, d f=5, p=.00$ & $x^{2}=63.6, d f=8, p=.00$ \\
\hline Decision errors & 1 & ns & $x^{2}=11.7, d f=5, p<.03$ & $x^{2}=35.7, d f=8, p=.00$ \\
\hline
\end{tabular}

Notes. HFACS = human factors analysis and classification system, $\mathrm{CRM}=\mathrm{crew}$ resource management.

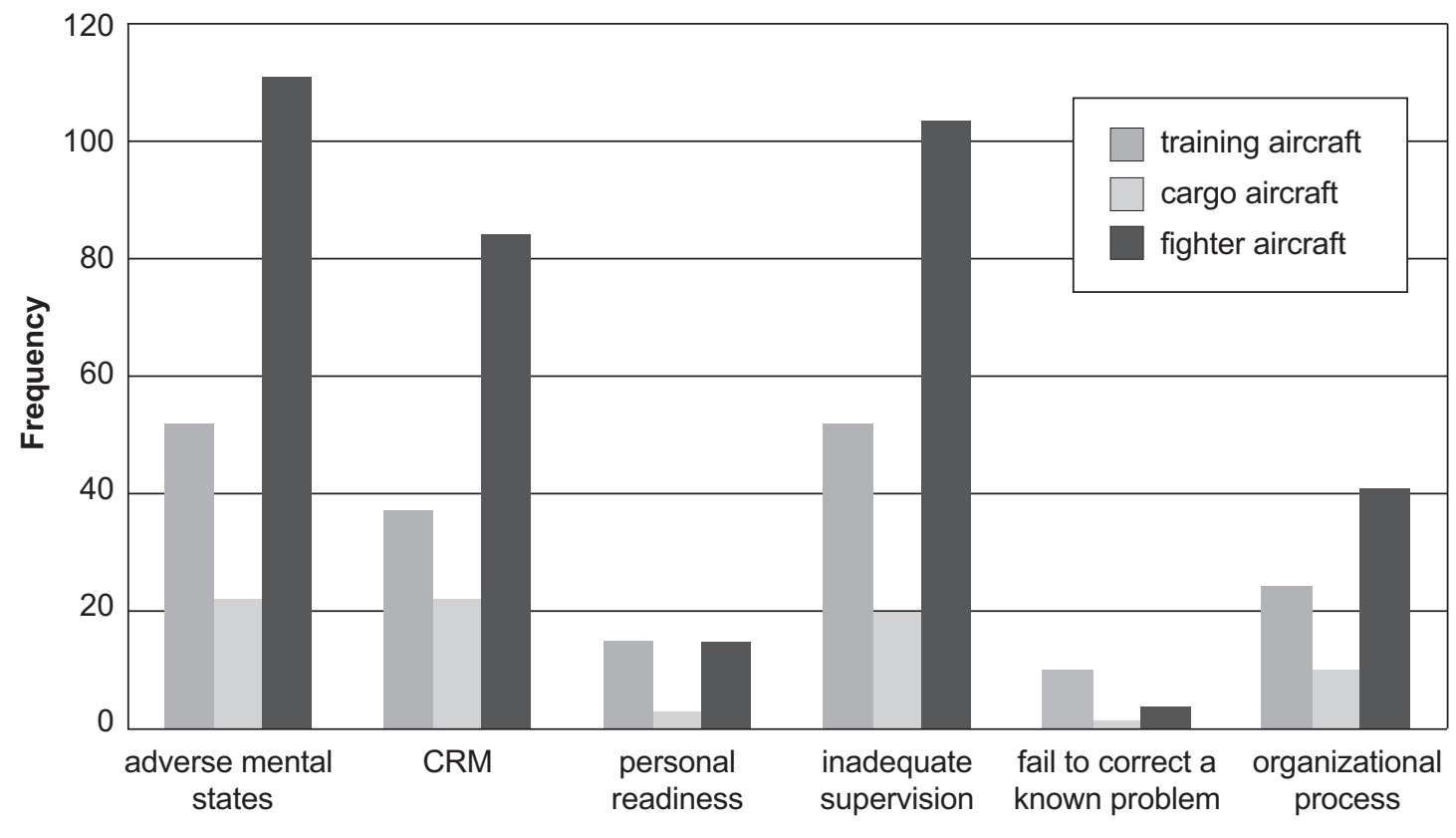

Category

Figure 2. Frequencies related to significant associations between HFACS categories and aircraft type (see Table 1). Notes. HFACS = human factors analysis and classification system, $\mathrm{CRM}=\mathrm{crew}$ resource management. 
TABLE 2. Significant Associations Between HFACS Categories and Aircraft Type

\begin{tabular}{|c|c|c|c|c|c|c|}
\hline \multirow[b]{3}{*}{ Aircraft Type } & \multirow{2}{*}{\multicolumn{2}{|c|}{$\begin{array}{c}\text { Adverse Mental States } \\
X^{2}=7.55, p=.020\end{array}$}} & \multicolumn{2}{|c|}{ CRM } & \multirow{2}{*}{\multicolumn{2}{|c|}{$\begin{array}{c}\text { Personal Readiness } \\
x^{2}=9.58, p=.010\end{array}$}} \\
\hline & & & $x^{2}=8.3$ & .010 & & \\
\hline & $\begin{array}{l}\text { Observed } \\
\text { Expected }\end{array}$ & $\begin{array}{c}\text { ASR } \\
p\end{array}$ & $\begin{array}{l}\text { Observed } \\
\text { Expected }\end{array}$ & $\begin{array}{c}\text { ASR } \\
p\end{array}$ & $\begin{array}{l}\text { Observed } \\
\text { Expected }\end{array}$ & $\begin{array}{c}\text { ASR } \\
p\end{array}$ \\
\hline Training & $\begin{array}{c}51 \\
40.1\end{array}$ & $\begin{array}{c}2.4 \\
<.020\end{array}$ & $\begin{array}{c}36 \\
31.8\end{array}$ & $\begin{array}{l}1.0 \\
\text { ns }\end{array}$ & $\begin{array}{l}13 \\
6.3\end{array}$ & $\begin{array}{l}3.1 \\
\text { ns }\end{array}$ \\
\hline Cargo & $\begin{array}{c}23 \\
20.2\end{array}$ & $\begin{array}{l}0.8 \\
\text { ns }\end{array}$ & $\begin{array}{c}24 \\
16.1\end{array}$ & $\begin{array}{c}2.5 \\
<.020\end{array}$ & $\begin{array}{c}2 \\
3.2\end{array}$ & $\begin{array}{c}-0.7 \\
\mathrm{~ns}\end{array}$ \\
\hline Fighter & $\begin{array}{c}110 \\
123.6 \\
\end{array}$ & $\begin{array}{l}-2.7 \\
<.010\end{array}$ & $\begin{array}{c}86 \\
98.1 \\
\end{array}$ & $\begin{array}{l}-2.5 \\
<.020\end{array}$ & $\begin{array}{c}14 \\
19.5 \\
\end{array}$ & $\begin{array}{l}-2.2 \\
<.050\end{array}$ \\
\hline
\end{tabular}

\begin{tabular}{|c|c|c|c|c|c|c|}
\hline \multirow[b]{3}{*}{ Aircraft Type } & \multirow{2}{*}{\multicolumn{2}{|c|}{$\begin{array}{l}\text { Inadequate Supervision } \\
\qquad X^{2}=8.28, p=.010\end{array}$}} & \multirow{2}{*}{\multicolumn{2}{|c|}{$\begin{array}{c}\begin{array}{c}\text { Fail to Correct } \\
\text { a Known Problem }\end{array} \\
X^{2}=20.69, p=.000\end{array}$}} & \multirow{2}{*}{\multicolumn{2}{|c|}{$\begin{array}{c}\text { Organizational Process } \\
\qquad x^{2}=7.74, p=.020\end{array}$}} \\
\hline & & & & & & \\
\hline & $\begin{array}{l}\text { Observed } \\
\text { Expected }\end{array}$ & $\begin{array}{c}\text { ASR } \\
p\end{array}$ & $\begin{array}{l}\text { Observed } \\
\text { Expected }\end{array}$ & $\begin{array}{c}\text { ASR } \\
p\end{array}$ & $\begin{array}{l}\text { Observed } \\
\text { Expected }\end{array}$ & $\begin{array}{c}\text { ASR } \\
p \\
\end{array}$ \\
\hline Training & $\begin{array}{c}51 \\
38.6\end{array}$ & $\begin{array}{l}2.8 \\
\mathrm{~ns}\end{array}$ & $\begin{array}{c}9 \\
2.6\end{array}$ & $\begin{array}{l}4.5 \\
\mathrm{~ns}\end{array}$ & $\begin{array}{c}25 \\
16.6\end{array}$ & $\begin{array}{c}2.5 \\
<.020\end{array}$ \\
\hline Cargo & $\begin{array}{c}20 \\
19.5\end{array}$ & $\begin{array}{l}0.2 \\
\text { ns }\end{array}$ & $\begin{array}{c}1 \\
1.3\end{array}$ & $\begin{array}{c}-0.3 \\
\mathrm{~ns}\end{array}$ & $\begin{array}{l}10 \\
8.4\end{array}$ & $\begin{array}{l}0.7 \\
\text { ns }\end{array}$ \\
\hline Fighter & $\begin{array}{c}106 \\
118.9\end{array}$ & $\begin{array}{l}-2.6 \\
<.010\end{array}$ & $\begin{array}{c}2 \\
8.1\end{array}$ & $\begin{array}{c}-3.8 \\
\mathrm{~ns}\end{array}$ & $\begin{array}{c}41 \\
51.1\end{array}$ & $\begin{array}{l}-2.7 \\
<.010\end{array}$ \\
\hline
\end{tabular}

Notes. HFACS = human factors analysis and classification system, CRM = crew resource management, ASR $=$ adjusted standardized residual.

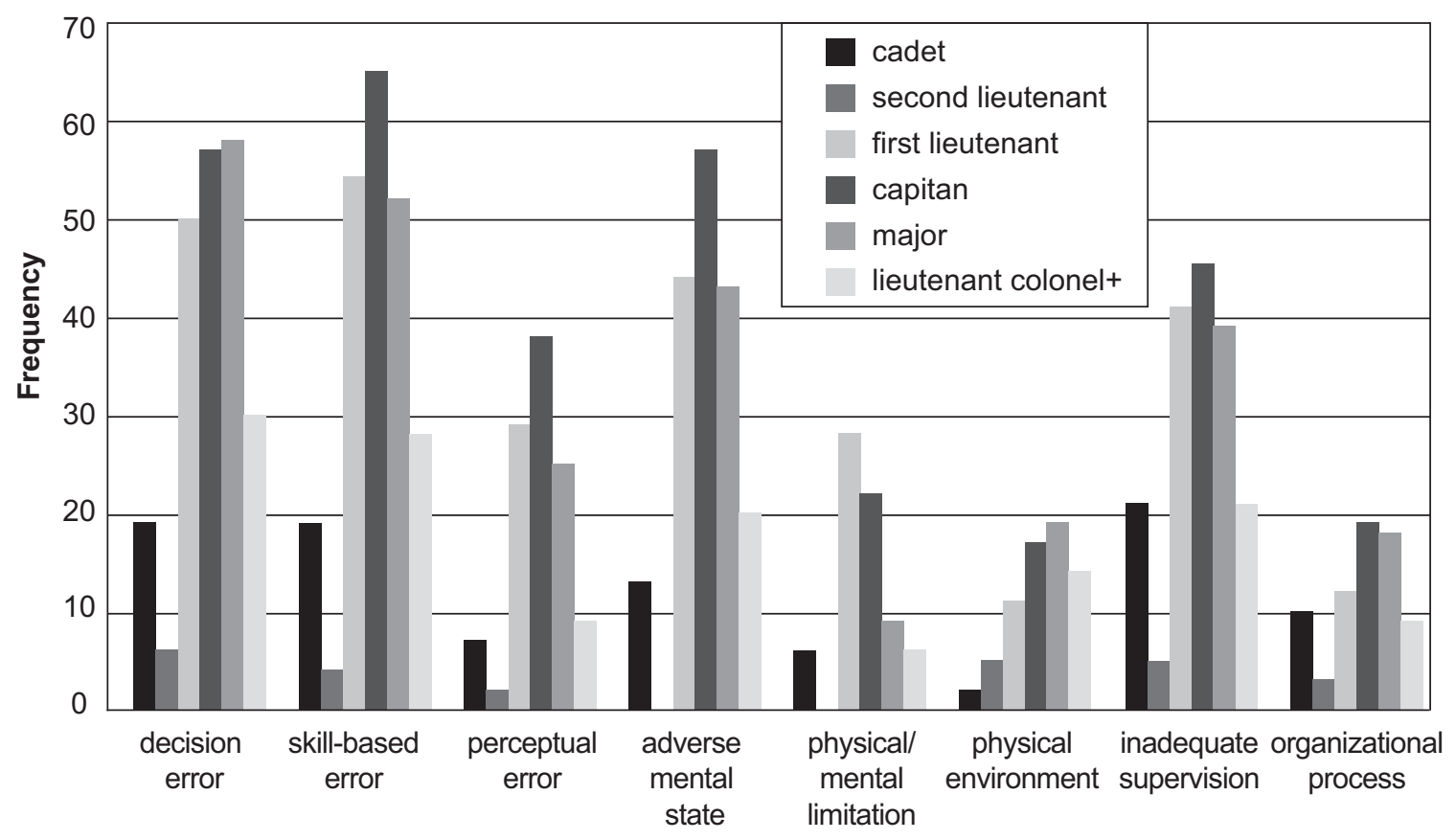

Category

Figure 3. Frequencies related to significant associations between HFACS categories and rank of the pilot involved in the accident (see Table 1). Notes. HFACS = human factors analysis and classification system, lieutenant colonel+ = lieutenant colonel or above. 
TABLE 3. Significant associations between HFACS categories and Pilots' Rank

\begin{tabular}{|c|c|c|c|c|c|c|c|c|}
\hline \multirow[b]{3}{*}{ Pilots' Rank } & \multicolumn{2}{|c|}{$\begin{array}{l}\text { Decision } \\
\text { Error }\end{array}$} & \multicolumn{2}{|c|}{$\begin{array}{l}\text { Skill-Based } \\
\text { Errors }\end{array}$} & \multicolumn{2}{|c|}{$\begin{array}{l}\text { Perceptual } \\
\text { Error }\end{array}$} & \multirow{2}{*}{\multicolumn{2}{|c|}{$\begin{array}{c}\text { Adverse Mental States } \\
\mathrm{X}^{2}=21.728, p<.005\end{array}$}} \\
\hline & \multicolumn{2}{|c|}{$x^{2}=14.812, p<.050$} & \multicolumn{2}{|c|}{$x^{2}=22.157, p<.005$} & \multicolumn{2}{|c|}{$x^{2}=14.005, p<.050$} & & \\
\hline & $\begin{array}{l}\text { Observed } \\
\text { Expected }\end{array}$ & $\begin{array}{c}\text { ASR } \\
p\end{array}$ & $\begin{array}{l}\text { Observed } \\
\text { Expected }\end{array}$ & $\begin{array}{c}\text { ASR } \\
p\end{array}$ & $\begin{array}{l}\text { Observed } \\
\text { Expected }\end{array}$ & $\begin{array}{c}\text { ASR } \\
p\end{array}$ & $\begin{array}{l}\text { Observed } \\
\text { Expected }\end{array}$ & $\begin{array}{c}\text { ASR } \\
p\end{array}$ \\
\hline Cadet & $\begin{array}{c}19 \\
13.7\end{array}$ & $\begin{array}{l}2.0 \\
<.050\end{array}$ & $\begin{array}{c}19 \\
13.8\end{array}$ & $\begin{aligned} & 2.0 \\
< & .050\end{aligned}$ & $\begin{array}{l}7 \\
6.8\end{array}$ & $\begin{array}{l}0.1 \\
\mathrm{~ns}\end{array}$ & $\begin{array}{c}13 \\
11.0\end{array}$ & $\begin{array}{l}0.8 \\
\mathrm{~ns}\end{array}$ \\
\hline $\begin{array}{l}\text { Second } \\
\text { lieutenant }\end{array}$ & $\begin{array}{c}6 \\
4.6\end{array}$ & $\begin{array}{l}0.9 \\
\text { ns }\end{array}$ & $\begin{array}{c}4 \\
4.6\end{array}$ & $\begin{array}{c}-0.4 \\
\mathrm{~ns}\end{array}$ & $\begin{array}{c}2 \\
2.3\end{array}$ & $\begin{array}{c}-0.2 \\
\mathrm{~ns}\end{array}$ & $\begin{array}{c}0 \\
3.7\end{array}$ & $\begin{array}{l}-2.4 \\
<.020\end{array}$ \\
\hline First lieutenant & $\begin{array}{l}50 \\
40.5\end{array}$ & $\begin{array}{c}2.2 \\
<.050\end{array}$ & $\begin{array}{l}54 \\
0.9\end{array}$ & $\begin{array}{l}3.1 \\
<.002\end{array}$ & $\begin{array}{c}29 \\
20.3\end{array}$ & $\begin{array}{l}2.4 \\
<.020\end{array}$ & $\begin{array}{c}44 \\
32.6\end{array}$ & $\begin{array}{l}2.8 \\
<.005\end{array}$ \\
\hline Captain & $\begin{array}{c}57 \\
63.3\end{array}$ & $\begin{array}{c}-1.3 \\
\mathrm{~ns}\end{array}$ & $\begin{array}{c}65 \\
63.9\end{array}$ & $\begin{array}{l}0.2 \\
\text { ns }\end{array}$ & $\begin{array}{c}38 \\
31.7\end{array}$ & $\begin{array}{l}1.5 \\
\mathrm{~ns}\end{array}$ & $\begin{array}{l}57 \\
50.9\end{array}$ & $\begin{array}{l}1.3 \\
\text { ns }\end{array}$ \\
\hline Major & $\begin{array}{l}58 \\
66.9\end{array}$ & $\begin{array}{l}-1.6 \\
\mathrm{~ns}\end{array}$ & $\begin{array}{c}52 \\
66.6\end{array}$ & $\begin{array}{l}-2.9 \\
<.005\end{array}$ & $\begin{array}{c}25 \\
33.0\end{array}$ & $\begin{array}{l}-1.9 \\
\mathrm{~ns}\end{array}$ & $\begin{array}{c}43 \\
53.1\end{array}$ & $\begin{array}{l}-2.1 \\
<.050\end{array}$ \\
\hline $\begin{array}{c}\text { Lieutenant } \\
\text { colonel+ }\end{array}$ & $\begin{array}{c}30 \\
29.2\end{array}$ & $\begin{array}{c}-0.3 \\
\mathrm{~ns}\end{array}$ & $\begin{array}{c}25 \\
29.4\end{array}$ & $\begin{array}{c}-1.2 \\
\mathrm{~ns}\end{array}$ & $\begin{array}{c}8 \\
14.6\end{array}$ & $\begin{array}{l}-2.1 \\
<.050\end{array}$ & $\begin{array}{c}18 \\
23.5\end{array}$ & $\begin{array}{c}-1.5 \\
\mathrm{~ns}\end{array}$ \\
\hline
\end{tabular}

\begin{tabular}{|c|c|c|c|c|c|c|c|c|}
\hline \multirow[b]{3}{*}{ Pilots' Rank } & \multicolumn{2}{|c|}{$\begin{array}{c}\text { Physical/Mental } \\
\text { Limitation }\end{array}$} & \multicolumn{2}{|c|}{ Physical Environment } & \multicolumn{2}{|c|}{$\begin{array}{l}\text { Inadequate } \\
\text { Supervision }\end{array}$} & \multicolumn{2}{|c|}{$\begin{array}{c}\text { Organizational } \\
\text { Process }\end{array}$} \\
\hline & \multicolumn{2}{|c|}{$x^{2}=33.298, p<.001$} & \multicolumn{2}{|c|}{$x^{2}=16.221, p<.050$} & \multicolumn{2}{|c|}{$x^{2}=31.797, p<.001$} & \multicolumn{2}{|c|}{$x^{2}=17.105, p<.050$} \\
\hline & $\begin{array}{l}\text { Observed } \\
\text { Expected }\end{array}$ & $\begin{array}{c}\text { ASR } \\
p\end{array}$ & $\begin{array}{l}\text { Observed } \\
\text { Expected }\end{array}$ & $\begin{array}{c}\text { ASR } \\
p\end{array}$ & $\begin{array}{l}\text { Observed } \\
\text { Expected }\end{array}$ & $\begin{array}{c}\text { ASR } \\
p\end{array}$ & $\begin{array}{l}\text { Observed } \\
\text { Expected }\end{array}$ & $\begin{array}{c}\text { ASR } \\
p\end{array}$ \\
\hline Cadet & $\begin{array}{l}6 \\
4.4\end{array}$ & $\begin{array}{l}0.8 \\
\text { ns }\end{array}$ & $\begin{array}{c}2 \\
4.2\end{array}$ & $\begin{array}{c}-1.2 \\
\mathrm{~ns}\end{array}$ & $\begin{array}{l}21 \\
10.7\end{array}$ & $\begin{array}{l}4.1 \\
<.001\end{array}$ & $\begin{array}{l}10 \\
4.4\end{array}$ & $\begin{array}{l}3.0 \\
<.002\end{array}$ \\
\hline $\begin{array}{l}\text { Second } \\
\text { lieutenant }\end{array}$ & $\begin{array}{c}0 \\
1.5\end{array}$ & $\begin{array}{c}-1.3 \\
\mathrm{~ns}\end{array}$ & $\begin{array}{l}5 \\
1.4\end{array}$ & $\begin{array}{l}3.3 \\
<.001\end{array}$ & $\begin{array}{c}5 \\
3.6\end{array}$ & $\begin{array}{l}1.0 \\
\mathrm{~ns}\end{array}$ & $\begin{array}{l}3 \\
1.5\end{array}$ & $\begin{array}{l}1.4 \\
\text { ns }\end{array}$ \\
\hline First lieutenant & $\begin{array}{l}28 \\
13.1\end{array}$ & $\begin{array}{l}4.9 \\
<.001\end{array}$ & $\begin{array}{c}11 \\
12.5\end{array}$ & $\begin{array}{c}-0.5 \\
\mathrm{~ns}\end{array}$ & $\begin{array}{c}41 \\
31.7\end{array}$ & $\begin{array}{l}2.3 \\
<.020\end{array}$ & $\begin{array}{c}12 \\
13.1\end{array}$ & $\begin{array}{c}-0.4 \\
\mathrm{~ns}\end{array}$ \\
\hline Captain & $\begin{array}{c}22 \\
20.4\end{array}$ & $\begin{array}{l}0.4 \\
\text { ns }\end{array}$ & $\begin{array}{c}17 \\
19.6\end{array}$ & $\begin{array}{c}-0.7 \\
\mathrm{~ns}\end{array}$ & $\begin{array}{c}45 \\
49.5\end{array}$ & $\begin{array}{c}-0.9 \\
\text { ns }\end{array}$ & $\begin{array}{c}19 \\
20.4\end{array}$ & $\begin{array}{c}-0.4 \\
\text { ns }\end{array}$ \\
\hline Major & $\begin{array}{c}9 \\
21.3\end{array}$ & $\begin{array}{l}-3.5 \\
<.001\end{array}$ & $\begin{array}{c}19 \\
20.4\end{array}$ & $\begin{array}{c}-0.4 \\
\mathrm{~ns}\end{array}$ & $\begin{array}{c}39 \\
51.6\end{array}$ & $\begin{array}{l}-2.6 \\
<.010\end{array}$ & $\begin{array}{c}18 \\
21.3\end{array}$ & $\begin{array}{c}-0.9 \\
\text { ns }\end{array}$ \\
\hline $\begin{array}{r}\text { Lieutenant } \\
\text { colonel+ }\end{array}$ & $\begin{array}{l}6 \\
9.4\end{array}$ & $\begin{array}{l}-1.3 \\
\mathrm{~ns}\end{array}$ & $\begin{array}{l}13 \\
9.0\end{array}$ & $\begin{array}{l}1.5 \\
\mathrm{~ns}\end{array}$ & $\begin{array}{c}18 \\
22.8\end{array}$ & $\begin{array}{c}-1.3 \\
\mathrm{~ns}\end{array}$ & $\begin{array}{l}8 \\
9.4\end{array}$ & $\begin{array}{c}-0.5 \\
\mathrm{~ns}\end{array}$ \\
\hline
\end{tabular}

Notes. HFACS = human factors analysis and classification system, ASR = adjusted standardized residual, lieutenant colonel+ = lieutenant colonel or above.

\subsection{HFACS Categories Versus Flight Phase}

Accidents happened during taxi before take-off phase in $36(6.9 \%)$ cases, in the take-off phase in $64(12.2 \%)$ cases, during climb out in $28(5.4 \%)$ instances. while maneuvering in the operational area in $200(38.2 \%)$ cases, during descent in 9 $(1.7 \%)$ instances, during approach in $55(10.5 \%)$ cases, while landing in $68(13 \%)$ cases and while taxiing after landing in $61(11.7 \%)$ instances.
At level 1 (unsafe acts of operators), the association of flight phase with decision errors and skilled-based errors was significant. The flight phase of landing was over-represented in both these categories of accident. Taxiing after landing was also over-represented in the frequency of skill-based errors. At level 2 (preconditions for unsafe acts), the associations between flight phase with adverse mental states, physical/ mental limitations and with CRM were all 
significant. The flight phase of operational area was over-represented in all these three categories of accidents. The flight phase of descent was also over-represented in the incidence of adverse mental states as an attributed factor. At level 3 (unsafe supervision), the association of flight phase with inadequate supervision was significant. The flight phase of landing was over-represented in the category of inadequate supervision. At level 4 (organizational influences), there was no significant association between flight phase and any category within the HFACS framework (see Tables 1 and 4, and Figure 4).

\section{DISCUSSION}

There were significant associations between specific categories in the HFACS framework and type of aircraft, rank and phase of flight.

\subsection{HFACS Categories Versus Aircraft Types}

The results showed that fighter aircraft had the highest frequency of accidents (342), followed by training (111) and cargo aircraft (56). All of these were uncorrected for exposure.

At level 1 (unsafe acts of operators), there were no significant associations with respect to aircraft type. At level 2 (preconditions for unsafe acts), the association of aircraft type with adverse mental states, CRM and personal readiness were significant. Training aircraft pilots were overrepresented in having adverse mental states and personal readiness as causal factors. Shappell and Weigmann suggest that adverse mental states encompasses those mental conditions that affect performance, such as a loss of situational awareness, task fixation, distraction and mental fatigue as a result of stress $[5,8]$. It is perhaps not too surprising that such factors were implicated in accidents involving training aircraft (and, hence, trainee pilots). Inappropriate attitudes, such as overconfidence, complacency and misplaced motivation, are included in this category. Again, those are attitudes which may be prevalent in cadet pilots. Personal readiness failures occur when pilots are not appropriately prepared for the flight that they are about to undertake. This can

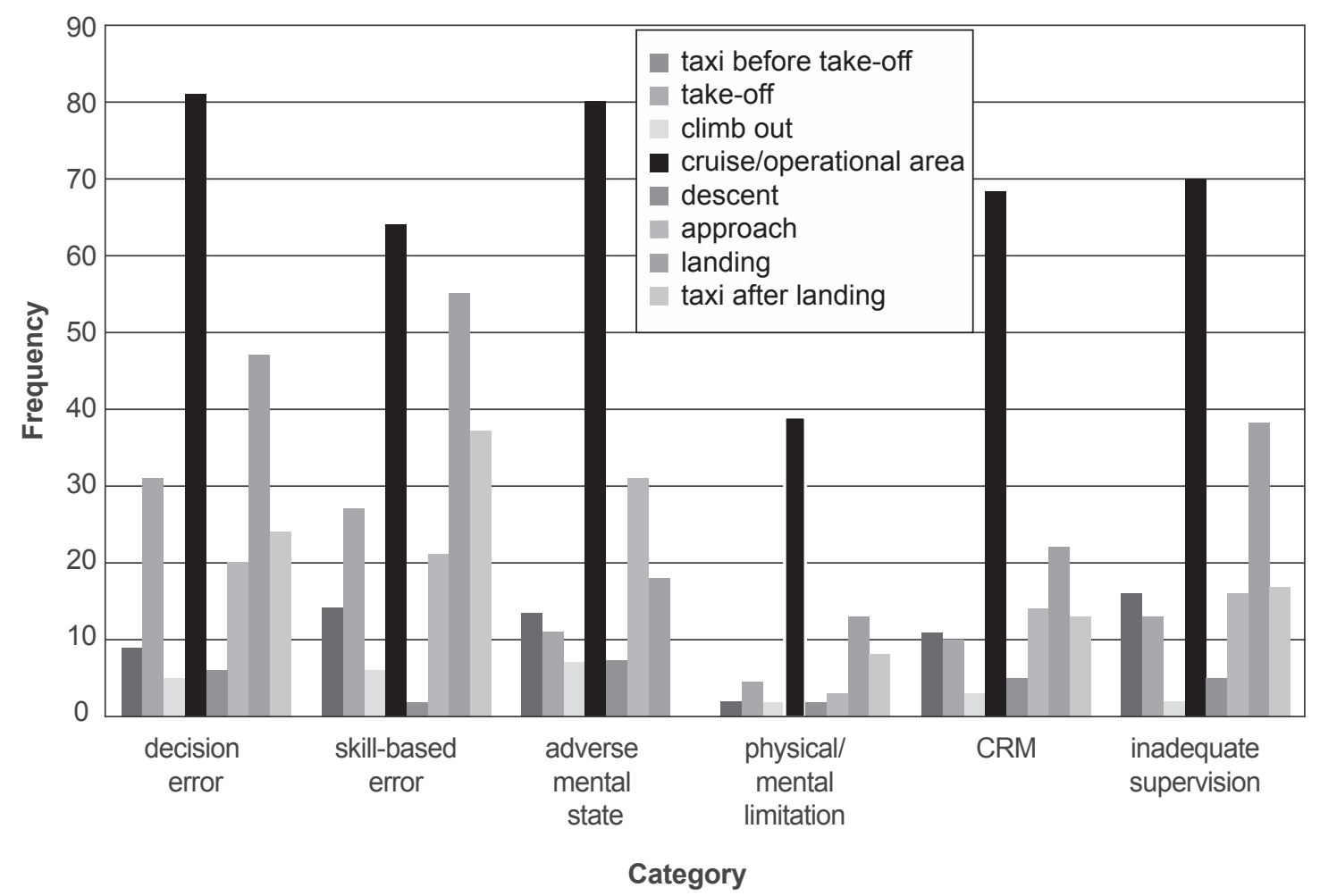

Figure 4. Frequencies related to significant associations between HFACS categories and flight phase (see Table 1). Notes. HFACS = human factors analysis and classification system. 
TABLE 4. Significant Associations Between HFACS Categories and Flight Phase

\begin{tabular}{|c|c|c|c|c|c|c|}
\hline \multirow[b]{3}{*}{ Flight Phase } & \multicolumn{2}{|c|}{ Decision Error } & \multicolumn{2}{|c|}{ Skill-Based Error } & \multicolumn{2}{|c|}{ Adverse Mental State } \\
\hline & \multicolumn{2}{|c|}{$x^{2}=35.767, p<.001$} & \multicolumn{2}{|c|}{$x^{2}=63.696, p<.001$} & \multicolumn{2}{|c|}{$x^{2}=25.757, p<.001$} \\
\hline & $\begin{array}{l}\text { Observed } \\
\text { Expected }\end{array}$ & $\begin{array}{c}\text { ASR } \\
p \\
\end{array}$ & $\begin{array}{l}\text { Observed } \\
\text { Expected }\end{array}$ & $\begin{array}{c}\text { ASR } \\
p \\
\end{array}$ & $\begin{array}{l}\text { Observed } \\
\text { Expected }\end{array}$ & $\begin{array}{c}\text { ASR } \\
p \\
\end{array}$ \\
\hline Taxi before take-off & $\stackrel{9}{15.8}$ & $\begin{array}{l}-2.4 \\
<.020\end{array}$ & $\begin{array}{c}14 \\
16.0\end{array}$ & $\begin{array}{c}-0.7 \\
\mathrm{~ns}\end{array}$ & $\begin{array}{l}13 \\
13\end{array}$ & $\begin{array}{c}0 \\
\text { ns }\end{array}$ \\
\hline Take-off & $\begin{array}{c}31 \\
28.0\end{array}$ & $\begin{array}{l}0.8 \\
\text { ns }\end{array}$ & $\begin{array}{c}27 \\
28.4\end{array}$ & $\begin{array}{c}-0.4 \\
\mathrm{~ns}\end{array}$ & $\begin{array}{c}11 \\
23.1\end{array}$ & $\begin{array}{l}-3.4 \\
<.001\end{array}$ \\
\hline Climb out & $\begin{array}{c}5 \\
11.4\end{array}$ & $\begin{array}{l}-2.6 \\
<.010\end{array}$ & $\begin{array}{c}6 \\
11.5\end{array}$ & $\begin{array}{l}-2.2 \\
<.050\end{array}$ & $\begin{array}{l}7 \\
9.4\end{array}$ & $\begin{array}{l}-1.0 \\
\text { ns }\end{array}$ \\
\hline $\begin{array}{l}\text { Cruise/operational } \\
\text { area }\end{array}$ & $\begin{array}{l}81 \\
84.1\end{array}$ & $\begin{array}{l}-0.6 \\
\mathrm{~ns}\end{array}$ & $\begin{array}{c}64 \\
85.2\end{array}$ & $\begin{array}{l}-3.9 \\
<.001\end{array}$ & $\begin{array}{l}80 \\
69.4\end{array}$ & $\begin{array}{l}2.0 \\
<.050\end{array}$ \\
\hline Descent & $\begin{array}{c}6 \\
3.9\end{array}$ & $\begin{array}{l}1.4 \\
\mathrm{~ns}\end{array}$ & $\begin{array}{l}2 \\
4\end{array}$ & $\begin{array}{c}-1.4 \\
\mathrm{~ns}\end{array}$ & $\begin{array}{l}7 \\
3.3\end{array}$ & $\begin{array}{l}2.6 \\
<.010\end{array}$ \\
\hline Approach & $\begin{array}{c}20 \\
22.8\end{array}$ & $\begin{array}{c}-0.8 \\
\text { ns }\end{array}$ & $\begin{array}{l}21 \\
23.1\end{array}$ & $\begin{array}{c}-0.6 \\
\mathrm{~ns}\end{array}$ & $\begin{array}{c}14 \\
18.8\end{array}$ & $\begin{array}{c}-0.5 \\
\mathrm{~ns}\end{array}$ \\
\hline Landing & $\begin{array}{c}47 \\
29.4\end{array}$ & $\begin{array}{l}4.7 \\
<.001\end{array}$ & $\begin{array}{c}55 \\
29.7\end{array}$ & $\begin{array}{l}6.7 \\
<.001\end{array}$ & $\begin{array}{c}31 \\
24.2\end{array}$ & $\begin{array}{l}1.9 \\
\mathrm{~ns}\end{array}$ \\
\hline Taxi after landing & $\begin{array}{c}24 \\
26.7\end{array}$ & $\begin{array}{l}-0.7 \\
\mathrm{~ns}\end{array}$ & $\begin{array}{c}37 \\
27.1 \\
\end{array}$ & $\begin{array}{l}2.7 \\
<.010 \\
\end{array}$ & $\begin{array}{c}18 \\
22.1\end{array}$ & $\begin{array}{c}-1.2 \\
\mathrm{~ns}\end{array}$ \\
\hline
\end{tabular}

\begin{tabular}{|c|c|c|c|c|c|c|}
\hline \multirow[b]{3}{*}{ Flight Phase } & \multirow{2}{*}{\multicolumn{2}{|c|}{$\begin{array}{c}\text { Physical/Mental Limitation } \\
\qquad \mathrm{X}^{2}=17.553, p<.05\end{array}$}} & \multicolumn{2}{|c|}{ CRM } & \multirow{2}{*}{\multicolumn{2}{|c|}{$\begin{array}{l}\text { Inadequate Supervision } \\
x^{2}=34.685, p<=0.001\end{array}$}} \\
\hline & & & \multicolumn{2}{|c|}{$X^{2}=19.634, p<.05$} & & \\
\hline & $\begin{array}{l}\text { Observed } \\
\text { Expected }\end{array}$ & $\begin{array}{c}\text { ASR } \\
p\end{array}$ & $\begin{array}{l}\text { Observed } \\
\text { Expected }\end{array}$ & $\begin{array}{c}\text { ASR } \\
p\end{array}$ & $\begin{array}{l}\text { Observed } \\
\text { Expected }\end{array}$ & $\begin{array}{c}\text { ASR } \\
p\end{array}$ \\
\hline Taxi before take-off & $\begin{array}{c}2 \\
5.2\end{array}$ & $\begin{array}{l}-1.6 \\
\mathrm{~ns}\end{array}$ & $\begin{array}{c}11 \\
10.3\end{array}$ & $\begin{array}{l}0.3 \\
\text { ns }\end{array}$ & $\begin{array}{c}16 \\
12.5\end{array}$ & $\begin{array}{l}1.3 \\
\mathrm{~ns}\end{array}$ \\
\hline Take-off & $\begin{array}{c}4 \\
9.2\end{array}$ & $\begin{array}{l}-2.0 \\
<.050\end{array}$ & $\begin{array}{c}10 \\
18.4\end{array}$ & $\begin{array}{l}-2.5 \\
<.020\end{array}$ & $\begin{array}{c}13 \\
22.3\end{array}$ & $\begin{array}{l}-2.6 \\
<.010\end{array}$ \\
\hline Climb out & $\begin{array}{c}2 \\
3.7\end{array}$ & $\begin{array}{c}-1.0 \\
\mathrm{~ns}\end{array}$ & $\begin{array}{l}3 \\
7.5\end{array}$ & $\begin{array}{l}-2.0 \\
<.05\end{array}$ & $\begin{array}{l}2 \\
9\end{array}$ & $\begin{array}{l}-3.0 \\
<.002\end{array}$ \\
\hline $\begin{array}{l}\text { Cruise/operational } \\
\text { area }\end{array}$ & $\begin{array}{c}39 \\
27.5\end{array}$ & $\begin{array}{l}3.0 \\
<.002\end{array}$ & $\begin{array}{c}68 \\
55.1\end{array}$ & $\begin{array}{l}2.6 \\
<.010\end{array}$ & $\begin{array}{c}70 \\
66.8\end{array}$ & $\begin{array}{c}-0.6 \\
\mathrm{~ns}\end{array}$ \\
\hline Descent & $\begin{array}{c}2 \\
1.3\end{array}$ & $\begin{array}{l}0.7 \\
\text { ns }\end{array}$ & $\begin{array}{c}5 \\
2.6\end{array}$ & $\begin{array}{l}1.8 \\
\text { ns }\end{array}$ & $\begin{array}{l}5 \\
3.1\end{array}$ & $\begin{array}{l}1.3 \\
\text { ns }\end{array}$ \\
\hline Approach & $\begin{array}{l}3 \\
7.5\end{array}$ & $\begin{array}{l}-1.9 \\
\text { ns }\end{array}$ & $\begin{array}{c}14 \\
14.9\end{array}$ & $\begin{array}{l}-0.3 \\
\mathrm{~ns}\end{array}$ & $\begin{array}{c}16 \\
18.1\end{array}$ & $\begin{array}{c}-0.6 \\
\mathrm{~ns}\end{array}$ \\
\hline Landing & $\begin{array}{l}13 \\
9.6\end{array}$ & $\begin{array}{l}1.3 \\
\mathrm{~ns}\end{array}$ & $\begin{array}{l}22 \\
19.2\end{array}$ & $\begin{array}{l}0.8 \\
\text { ns }\end{array}$ & $\begin{array}{c}38 \\
23.3\end{array}$ & $\begin{array}{l}4.0 \\
<.001\end{array}$ \\
\hline Taxi after landing & $\begin{array}{c}8 \\
8.7\end{array}$ & $\begin{array}{l}-0.3 \\
\mathrm{~ns}\end{array}$ & $\begin{array}{c}13 \\
17.5\end{array}$ & $\begin{array}{c}-1.4 \\
\mathrm{~ns}\end{array}$ & $\begin{array}{c}17 \\
21.2\end{array}$ & $\begin{array}{c}-1.2 \\
\mathrm{~ns}\end{array}$ \\
\hline
\end{tabular}

Notes. HFACS = human factors analysis and classification system, CRM = crew resource management, ASR $=$ adjusted standardized residual.

include issues, such as mental fatigue as a result of gaining insufficient rest, inappropriate nutrition or fatigue as a result of excess physical training, all issues cadet pilots in a high-pressure training program are prone to.

Cargo aircraft pilots were over-represented in having CRM problems as the major causal factor as a result of this category of airplane being operated by multiple crew members. The $\chi^{2}$ test of association looks at the relative instance of HFACS causal frequencies, so this result should not be overinterpreted. Nevertheless, looking at the frequency of occurrence of CRM as a causal factor (24) versus the number of accidents 
involving cargo aircraft (56), there would still be evidence to suggest that CRM (inter- and intracockpit co-ordination, communication and leadership) could be improved.

At level 3 (unsafe supervision), the association of aircraft type with both inadequate supervision and failed to correct a known problem were significant. Training aircraft were again over-represented in both these categories. Both these categories are associated with organizational aspects of the provision of training, particularly the revision of training practices to reflect emerging requirements and the need to provide remedial training where necessary. These issues are particularly important in the operation of training aircraft.

Training aircraft accidents were also overrepresented in having the level-4 organizational process category as a factor. Given that poor supervisory practices were implicated in training aircraft accidents, it is not surprising that the related organizational process category, which includes the oversight of operational standards, was also involved. Previous studies have found strong statistical relationships between the high level of the organizational process category and the level-3 categories of inadequate supervision and failed to correct a known problem [34, 37].

The training aircraft have the highest usage in the RoC Air Force, hence, there is time pressure for maintenance and the regular airworthiness checks. Instructor pilots may also not have time to provide the appropriate amount of training/ supervision. Training aircraft are also operated by novice pilots, who may not be completely ready for solo flight. Fighters were generally under-represented in all the HFACS categories. Compared to trainee pilots, fighter pilots are relatively much more experienced and, hence, more capable to deal with demanding flight circumstances when flying solo.

\subsection{HFACS Categories Versus Pilots' Rank}

A pilot's rank is closely related to flying experience: senior officers have more flying hours than junior officers. The rank of cadet was significantly over-represented in having the categories of organizational process and inadequate supervision. This would suggest failures in the provision of training and the oversight of the training process. If instructor pilots do not provide proper training/supervision or monitoring of progress, sending a novice pilot (or a pilot new to the type of aircraft) solo, when he had not developed the necessary decision-making or psychomotor skills, may result in an accident. It is important to emphasize those accidents involving cadet pilots still had level-1 categories implicated in them (suggesting deficiencies in training provision in these areas); however, this particular analysis suggests that the effects of poor training oversight are particularly evident in trainee pilots early in their flying career. It was perhaps the junior cadet pilots' lack of experience and competence to deal with organizational influences that made them vulnerable to a higher level of managerial shortcomings.

Pilots with the rank of either second or first lieutenant were also novice pilots (with 200-500 flying hours) at the early stages of conversion from training aircraft to more advanced, higher performance combat aircraft. During this conversion period, there was an increased accident rate. The HFACS categories over-represented for these pilots were all associated with the development of more advanced flying skills (the level-1 categories of decision errors, skill-based errors and perceptual errors) and the stresses associated with a demanding training regime (level 2 of categories of adverse mental states and/or physical/ mental limitations). In the HFACS framework, decision errors are behaviors that proceed as intended; however, the plan upon which they are predicated is inadequate or inappropriate for the situation. They are subdivided into three further categories: procedural errors, poor choices and problem solving errors. Decision errors resulting from misdiagnosis of a situation (and, hence, poor subsequent choices) are common in highly time-critical situations, such as abnormal or emergency situations on takeoff or landing. Shappell and Weigmann included factors in the category of skill-based errors, such as the inadvertent use of flight controls, omitting steps in procedures or checklists, poor technique or overcontrolling the aircraft $[5,8]$. Perceptual errors encompassed factors, such as misjudging 
distance, altitude or airspeed. The level-2 category of adverse mental states in which pilots of this rank were over-represented covers the issues that junior pilots are prone to at the beginning of their operational flying career (see section 4.1). Physical/mental limitations occur when mission requirements exceed the pilot's capabilities. These normally apply to basic sensory and information processing limitations but may also be applied to pilots who simply do not have the aptitude for military aviation. This may still be a factor underlying the accidents of relatively inexperienced pilots new to flying a high performance aircraft.

The rank of lieutenant colonel (or above) was significantly associated with causal factors in the physical environment category. This HFACS category covers both the physical environment inside the aircraft (e.g., noise, vibration and temperature-all performance shaping factors known to predispose operators to making errors) and the environment outside the aircraft (meteorological conditions, light conditions or the terrain over which the aircraft is operating). The over-representation of pilots of this rank being associated with the physical environment and being implicated as a causal factor is probably attributable to the fact that senior pilots tend to hold the approvals to undertake more complex missions in adverse weather or over difficult terrain. In the RoC Air Force, rank is very highly associated with the number of flight hours accrued. While lower ranking officers with extensive flight time may also hold such approvals, it is far more likely that they will be undertaken by more senior ranks.

\subsection{HFACS Categories Versus Flight Phase}

There was a significant association between flight phase and six categories in the HFACS framework. At level 1 (unsafe acts of operators), both decision errors and skill-based errors were significantly associated with the landing flight phase. In the landing phase, precise psychomotor skills are required to control the aircraft and occasionally instant decisions and responses are necessary. This result would also be in accord with the number of accidents reported to training aircraft.
At level 2 (preconditions for unsafe acts), the categories of adverse mental states, physical/ mental limitation and CRM were significantly associated with the phase of flying in the operational area. Military tactical training, such as air combat tactics or low altitude tactics, places a high physical and mental demand on the pilots. Pilots need to be aware of the cognitive demands while flying in the operational area. Pilots are required to be in a heightened mental state to allow for rapid analysis of the situation followed by swift responses. They also need good CRM skills to deal with emergent risks and balance the priorities of safety versus accomplishing the mission.

At level 3 (unsafe supervision), inadequate supervision was again significantly associated with landing accidents (as it was with close pattern accidents and accidents involving training aircraft). This may again have been a result of instructors failing to provide adequate supervision, providing inappropriate instruction for landing or failing to track progress and/or provide suitable remedial training as a result.

\subsection{Identifying Training Requirements}

Inspection of the data in Figures 2-4 suggests that the principal pilot training requirements identified in this study is associated with the HFACS categories of decision errors, skill-based errors and CRM.

Some pilots in the RoC Air Force were of the opinion that CRM was only of benefit in civil aviation or multicrew operations and did not apply to single-seat fighter pilots. However, poor CRM was not only significantly associated with poor flying skills but was also significantly associated with poor decision-making. For example, there have been several midair crashes where the accident investigation concluded that poor communication and teamwork among pilots and air traffic control reduced situational awareness. As a result, pilots made inappropriate decisions and committed operational skill-based errors resulting in these catastrophes. Decision-making is a key component of CRM which encompasses problem definition/diagnosis, option generation, risk assessment/option choice and outcome review 
[25]. Aeronautical decision-making is trainable $[28,29,30,31]$ and such training, along with simulator practice, can produce better decisions [33]. CRM behaviors improve after appropriately targeted training [26] and can result in a substantial reduction in the accident rate [27].

\subsection{Organizational Issues in the Delivery of Pilot Training}

Many other HFACS categories were implicated in RoC Air Force accidents associated with either organizational processes, such as the design and oversight of training (level 3: unsafe supervision, specifically the categories of inadequate supervision and failed to correct a known problem) or the provision of the necessary infrastructure to assure the delivery of good training (level 4: organizational influences, specifically the category of organizational process).

The modern view of accident causation is that there is rarely a single cause to an accident. Accidents do not result solely from technical failures or human errors. The systemic view of accidents is that they result from organizational (latent) failures, distal errors and system mis-specifications, which lie dormant within the system but which serve to combine together with other factors to breach a system's defenses. As Reason observed, complex systems are designed, operated, maintained and managed by human beings, so it is not surprising that human decisions and actions at an organizational level are implicated in all accidents [23].

The analysis of accidents and incidents should be just one aspect of a continuous safety auditing process. However, it has to be emphasized that such reactive analyses alone are insufficient to ensure safety and should be supplemented with a proactive approach, i.e., identifying hazards and safety risks before they result in an accident $[23,38]$.

Reason's model of accident causation, upon which HFACS is based, commences at a point (organizationally) remote from the actual accident at the higher levels in the organization. The upper levels of management set priorities that subsequently have a secondary effect throughout the organization. These management decisions are more likely to be based around visible, rapid feedback (e.g., cost savings and efficiency increases), whereas safety gains always cost money, are unpredictable (prone to random fluctuations) and are often invisible. In this study, it was observed that the organizational process HFACS category, which includes the oversight of operational standards, was often implicated in accidents.

Management decisions are translated into practice at the supervisory level. Any deficiencies here have the direct effect of creating the psychological pre-cursors of unsafe acts. These function types include factors, such as poor tasking, deficient training, inadequate procedures, etc. Inadequate supervision and failed to correct a known problem were factors implicated in accidents both associated with the organizational aspects of the provision of training, particularly the revision of training practices, and the need to provide remedial training. To exemplify further, poor training may lead to psychological pre-cursors of error, such as high workload, misdiagnosis or the misperception of risks.

\section{CONCLUSION}

This investigation has demonstrated the usefulness of the HFACS framework to identify deficiencies in the content and delivery of training in the RoC Air Force (training needs) and ascertain organizational shortcomings in the infrastructure tasked with providing this training. HFACS has proved to be a valuable tool in supporting the initial stages of the training design process described by the IPISD [1]. It has successfully identified specific training gaps in the development of both cadet and junior (lieutenant) pilots underlying accidents in the RoC Air Force. This research draws a clear picture that supports Reason's model of active failure and latent conditions in the organization (upon which the HFACS framework is based) [4]. Fallible decisions of upperlevel command can directly affect the middle level of supervisory practices, creating preconditions for unsafe acts and impaired performance of pilots, leading to accidents. 


\section{REFERENCES}

1. Branson RK, Rayner GT, Cox L, Furman JP, King FJ, Hannum WH. Interservice procedures for instructional systems development: executive summary and model. Tallahasee, FL, USA: Center for Educational Technology, Florida State University; 1975. Retrieved January 31, 2013, from: http://www.dtic.mil/dtic/tr/ fulltext/u2/a019488.pdf.

2. Kirwan B, Ainsworth LK, editors. A guide to task analysis. Boca Raton, FL, USA: CRC Press; 1992.

3. Patrick J. Training. In: Tsang PS, Vidulich MA, editors. Principles and practice of aviation psychology. Mahwah, NJ, USA: Erlbaum; 2003. p. 397-434.

4. Reason JT. Human error. Cambridge, UK: Cambridge University Press; 1990.

5. Shappell SA, Wiegmann DA. A human error analysis of general aviation controlled flight into terrain accidents occurring between 1990-1998 (Report No. DOT/FAA/AM03/4). Washington, DC, USA: Federal Aviation Administration; 2003. Retrieved January 31, 2013, from: http://www.hf.faa. gov/docs/508/docs/cami/0304.pdf.

6. Wiegmann DA, Shappell SA. A human error approach to aviation accident analysis: the human factors analysis and classification system. Aldershot, UK: Ashgate; 2003.

7. Wiegmann DA, Shappell SA. Human factors analysis of postaccident data: applying theoretical taxonomies of human error. Int J Av Psychol. 1997;7(1):67-81.

8. Shappell SA, Wiegmann DA. The human factors analysis and classification systemHFACS (Report No. DOT/FAA/AM-00/7). Washington DC, USA: Federal Aviation Administration; 2000. Retrieved January 31, 2013, from: http://www.nifc. gov/fireInfo/fireInfo_documents/ humanfactors_classAnly.pdf.

9. Wiegmann DA, Shappell SA. Applying the human factors analysis and classification system to the analysis of commercial aviation accident data. In: Jensen RS, editor. Eleventh International Symposium on Aviation Psychology. Columbus, OH, USA: Ohio State University; 2001.
10. Wiegmann DA, Shappell SA. Human error perspectives in aviation. Int J Av Psychol. 2001;11(4):341-57.

11 Wiegmann DA, Shappell SA. Human error analysis of commercial aviation accidents: application of the human factors analysis and classification system (HFACS). Aviat Space Environ Med. 2001;72(11):1006-16.

12. Shappell SA, Wiegmann DA. Applying reason: the human factors analysis and classification system (HFACS). Human Factors and Aerospace Safety. 2001;1(1): 59-86.

13. Shappell SA, Wiegmann DA. HFACS analysis of military and civilian aviation accidents: a North American comparison. In: Proceedings of International Society of Air Safety Investigators, Australia, Queensland. Sterling, VA, USA. International Society of Air Safety Investigators; 2004. p. 2-8.

14. Dekker SWA. The re-invention of human error. Human Factors and Aerospace Safety. 2001;1(1):247-66.

15. Diehl A. The effectiveness of training programs for preventing aircrew error. In: Jensen RS, editor. Sixth International Symposium on Aviation Psychology. Columbus, $\mathrm{OH}, \mathrm{USA}$ : Ohio State University; 1991. vol. 2, p. 640-55.

16. Jensen RS. The boundaries of aviation psychology, human factors, aeronautical decision making, situation awareness, and crew resource management. Int J Av Psychol. 1994;7(4):259-67.

17. Klein GA. Analysis of situation awareness from critical incident reports. In: Endsley MR, Garland DJ, editors. Situation awareness analysis and measurement. Mahwah, NJ, USA: Erlbaum; 2000. p. 45-62.

18. Diehl A. Human performance/system safety issues in aircraft accident investigation and prevention. In: Jensen RS, editor. Fifth International Symposium on Aviation Psychology. Columbus, OH, USA: Ohio State University; 1989. vol. 2, p. 838-47.

19. Feggetter AJ. The development of an intelligent human factors data base as an aid for the investigation of aircraft accidents. In: Jensen RS, editor. Sixth International Symposium on Aviation 
Psychology. Columbus, OH, USA: Ohio State University; 1991. vol. 2, p. 324-9.

20. Harle PG. investigation of human factors: the link to accident prevention. In: McDonald N, Johnston R, Fuller R, editors. Applications of psychology to the aviation system. Aldershot, UK: Ashgate; 1985. p. 101-7.

21. Hollnagel E. Cognitive reliability and error analysis method (CREAM). Oxford, UK: Elsevier Science; 1998.

22. Hunter DR, Baker RM. Reducing accidents among general aviation pilots through a national aviation safety program. In: Hayward BJ, Lowe AR, editors. The Fourth Australian Aviation Psychology Symposium. Aldershot, UK: Ashgate; 2000. vol. 1, p. 63-72.

23. Reason J. Managing the risks of organizational accidents. Aldershot, UK: Ashgate; 1997.

24. Joint Aviation Authorities (JAA). Crew resource management-flight crew. Temporary guidance leaflet 5 (JAR-OPS). Administrative and guidance material (Section 4 - Operations). Hoofdorp, The Netherlands: JAA; 1998.

25. van Avermaete JAG. NOTECHS: Nontechnical skill evaluation in JAR-FCL (NLR-TP-98518). Amsterdam, The Netherlands: National Aerospace Laboratory; 2008. Retrieved January 31, 2013, from: http://www.simulationaustralia. org.au/archive/simtect/2005/NOTECHS_ report.pdf.

26. Ikomi PA, Boehm-Davis D, Holt RW, Incalcaterra KA. Jump seat observations of advanced crew resource management (ACRM) Effectiveness. In: Jensen RS, Cox B, Callister JD, Lavis R, editors. Tenth International Symposium on Aviation Psychology. Columbus, OH, USA: Ohio State University; 1999. vol. 1, p. 292-7.

27. Diehl A. The effectiveness of training programs for preventing aircrew error. In: Jensen RS, editor. Sixth International Symposium on Aviation Psychology. Columbus, OH, USA: Ohio State University; 1991. vol. 2, p. 640-55.

28. Endsley MR. A survey of situation awareness requirements in air-to-air combat fighters. Int J Av Psychol. 1993; 3(2):157-68.

29. Klein GA. A recognition-primed decision (RPD) model of rapid decision making. In: Klein GA, Orasanu J, Calderwood R, Zsambok CE, editors. Decision making in action: models and methods. Norwood, NJ, USA: Ablex; 1993. p. 138-47.

30. Klein G. The current status of the naturalistic decision making framework. In: Flin R, Salas E, Strub M, Martin L, editors. Decision making under stress: emerging themes and applications. Aldershot, UK: Ashgate; 1997. p. 11-28.

31 Li WC, Harris D. The evaluation of the effect of a short aeronautical decisionmaking training program for military pilots. Int J Av Psychol. 2008;18(2):135-52.

32. Buch G, Diehl A. An investigation of the effectiveness of pilot judgment training. Hum Factors. 1984;26(5):557-64.

33. Connolly TJ, Blackwell BB, Lester LF. A simulator-based approach to training in aeronautical decision making. Aviat Space Environ Med. 1989;60(1):50-2.

34. Li WC, Harris D. Pilot error and its relationship with higher organizational levels: HFACS analysis of 523 accidents. Aviat Space Environ Med. 2006;77(10): 1056-61.

35. Landis JR, Koch GG. The measurement of observer agreement for categorical data. Biometrics. 1977;33(1):159-74.

36. Gaur D. Human factors analysis and classification system applied to civil aircraft accidents in India. Aviat Space Environ Med. 2005;76(5):501-5.

37. Li WC, Harris D, Yu CS. Routes to failure: analysis of 41 civil aviation accidents from the Republic of China using the human factors analysis and classification system. Acc Anal Prev. 2008;40(2):426-34.

38. International Civil Aviation Organization (ICAO). Safety management manual. 2nd ed. (Document No. 9859, AN/474). Montreal, QC, Canada: ICAO; 2009. Retrieved January 31, 2013, from: http:// www.icao.int/safety/ism/Guidance $\% 20$ Materials/DOC_9859_FULL_EN.pdf. 